This is a preprint of: "Global phase portraits of quadratic systems with a complex ellipse as invariant algebraic curve", Jaume Llibre, Clàudia Valls, Acta Math. Sinica (N.S.), vol. 34(801), 11 pages, 2018.

DOI: $[10.1007 / \mathrm{s} 10114-017-5478-y]$

\title{
GLOBAL PHASE PORTRAITS OF QUADRATIC SYSTEMS WITH A COMPLEX ELLIPSE AS INVARIANT ALGEBRAIC CURVE
}

\author{
JAUME LLIBRE AND CLAUDIA VALLS
}

\begin{abstract}
In this paper we study a new class of quadratic systems and classify all its phase portraits. More precisely, we characterize the class of all quadratic polynomial differential systems in the plane having a complex ellipse $x^{2}+y^{2}+1=0$ as invariant algebraic curve. We provide all the different topological phase portraits that this class exhibits in the Poincaré disc.
\end{abstract}

\section{INTRODUCTION AND STATEMENT OF THE MAIN RESULTS}

A planar polynomial differential system is a differential system of the form

$$
\begin{aligned}
& \dot{x}=P(x, y), \\
& \dot{y}=Q(x, y),
\end{aligned}
$$

where $P$ and $Q$ are real polynomials. The polynomial differential system (1) has degree $n$, if $n$ is the maximum of the degrees of the polynomials $P$ and $Q$. A polynomial differential system of degree 2 is called simply a quadratic system. The dot in system (1) denotes the derivative with respect to the independent variable $t$.

Many families of quadratic systems have been studied, and for them all the possible global topological phase portraits have been classified. One of the first of these classes studied was the classification of the quadratic centers and their first integrals which started with the works of Dulac [6], Kapteyn [9, 10], Bautin [3], Lunkevich and Sibirskii [14], Schlomiuk [21], Żołądek [26], Ye and Ye [24], Artés, Llibre and Vulpe $[2], \ldots$ The class of the homogeneous quadratic systems, see Lyagina [15], Markus [16], Korol [11], Sibirskii and Vulpe [22], Newton [19], Date [5] and Vdovina [23],... Another class is the one formed by the Hamiltonian quadratic systems, see Artés and Llibre [1], Kalin and Vulpe [8], and Artés, Llibre and Vulpe [2].

2010 Mathematics Subject Classification. Primary 34C05.

Key words and phrases. quadratic system, complex ellipse, invariant algebraic curves, phase portrait. 
In this paper we want to study a new class of quadratic systems and classify all its phase portraits. More precisely we analyze the class of all quadratic polynomial differential systems having a complex invariant ellipse, that after an affine transformation it can be written as $x^{2}+$ $y^{2}+1=0$. The family of quadratic systems having a real invariant ellipse have been studied by Qin Yuan-Xun [25] in 1958.

Our first result is to provide a normal form for all quadratic polynomial differential systems having a complex invariant ellipse $x^{2}+y^{2}+1=$ 0 as an invariant algebraic curve.

Proposition 1. A planar polynomial differential system of degree 2 having a complex invariant ellipse $x^{2}+y^{2}+1=0$ as an invariant algebraic curve, after an affine change of coordinates, can be written as

$$
\begin{aligned}
& \dot{x}=\alpha\left(x^{2}+y^{2}+1\right)+y(a x+b y+c), \\
& \dot{y}=-x(a x+b y+c)
\end{aligned}
$$

where $\alpha, a, b, c \in \mathbb{R}$.

The proof of Proposition 1 is the same as the proof of the normal form of the quadratic system with a real invariant ellipse, see for more details the normal form given in system (12) of [13].

In the next theorem we present the topological classification of all the phase portraits in the Poincaré disc of planar polynomial differential systems of degree 2 having a complex invariant ellipse $x^{2}+y^{2}+1=$ 0 as an invariant algebraic curve. For a definition of the Poincaré compactification, the Poincaré disc and of topological equivalent phase portraits of a polynomial differential system in the Poincaré disc, see section 2 .

It is known that if a quadratic system has a limit cycle this surrounds a focus, see for more details [4]. Due to this fact we shall see that such quadratic systems have no limit cycles.

Theorem 2. Given a planar polynomial differential system of degree 2 having a complex ellipse $x^{2}+y^{2}+1=0$ as an invariant algebraic curve its phase portrait is topological equivalent to one of the 12 phase portraits of Figure 1.

Theorem 2 is proved in section 3 . 

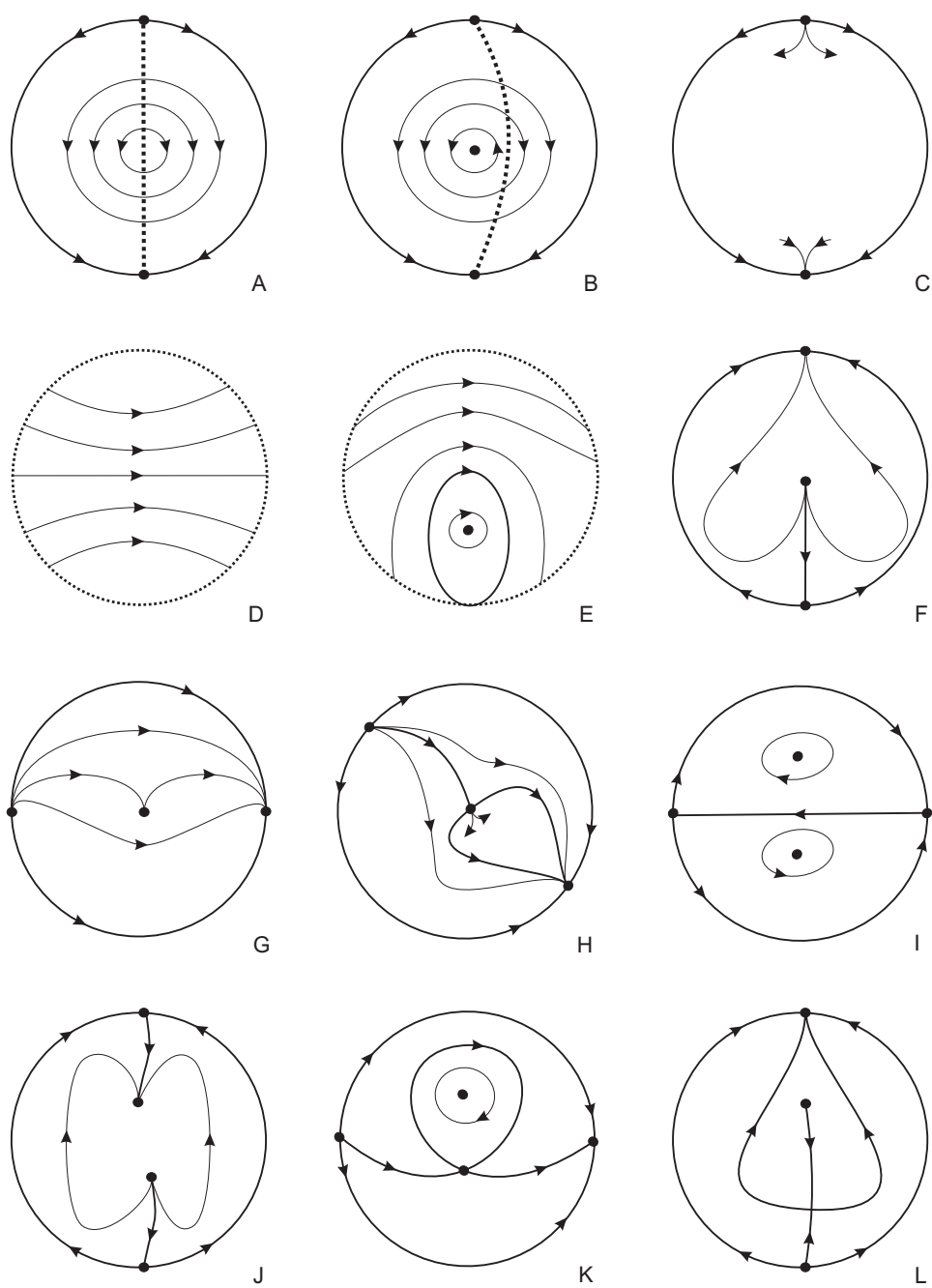

Figure 1. Phase portraits in the Poincaré disc of the quadratic systems having an invariant complex ellipse.

\section{BASIC RESULTS}

2.1. Poincaré compactification. The planar polynomial vector field of degree $n$ associated to the polynomial differential system (1) of degree $n$ is

$$
\mathcal{X}=P(x, y) \frac{\partial}{\partial x}+Q(x, y) \frac{\partial}{\partial y} .
$$


The Poincaré compactified vector field $p(\mathcal{X})$ associated to $\mathcal{X}$ is an analytic vector field on $\mathbb{S}^{2}$ constructed as follows (see, for more details Chapter 5 of $[7])$.

Let $\mathbb{S}^{2}=\left\{y=\left(y_{1}, y_{2}, y_{3}\right) \in \mathbb{R}^{3}: y_{1}^{2}+y_{2}^{2}+y_{3}^{2}=1\right\}$ (the Poincaré sphere) and $T_{y} \mathbb{S}^{2}$ be the tangent plane to $\mathbb{S}^{2}$ at point $y$. The tangent plane $T_{(0,0,1)} \mathbb{S}^{2}$ is identified with the plane $\mathbb{R}^{2}$ where we have the polynomial vector field $\mathcal{X}$. Consider the central projections $f_{ \pm}: T_{(0,0,1)} \mathbb{S}^{2} \rightarrow$ $\mathbb{S}^{2}$. These two maps define two copies of $\mathcal{X}$, one $\mathcal{X}^{+}$in the northern hemisphere and the other $\mathcal{X}^{-}$in the southern hemisphere. Denote by $\mathcal{X}^{\prime}=\mathcal{X}^{+} \cup \mathcal{X}^{-}$the vector field defined on $\mathbb{S}^{2}$ except on its equator $\mathbb{S}^{1}=\left\{y \in \mathbb{S}^{2}: y_{3}=0\right\}$. The infinity of $\mathbb{R}^{2}$ is identified with the equator $\mathbb{S}^{1}$. The extension of $\mathcal{X}^{\prime}$ to a vector field $P(\mathcal{X})$ on $\mathbb{S}^{2}$ (including $\mathbb{S}^{1}$ ) is the unique analytic extension of $y_{3}^{n-1} \mathcal{X}^{\prime}$ to $\mathbb{S}^{2}$. Knowing the behaviour of $p(\mathcal{X})$ in a neighborhood of $\mathbb{S}^{1}$, we know the behaviour of $\mathcal{X}$ near the infinity. The Poincaré compactification is such that $\mathbb{S}^{1}$ is invariant under the flow of $p(\mathcal{X})$.

The Poincaré disc, denoted by $\mathbb{D}$, is the projection of the closed northern hemisphere of $\mathbb{S}^{2}$ on $y_{3}=0$ under $\left(y_{1}, y_{2}, y_{3}\right) \longmapsto\left(y_{1}, y_{2}\right)$.

Two polynomial vector fields $\mathcal{X}$ and $\mathcal{Y}$ on $\mathbb{R}^{2}$ are topologically equivalent if there is a homeomorphism on $\mathbb{S}^{2}$ carrying orbits of the flow induced by $p(\mathcal{X})$ into orbits of the flow induced by $p(\mathcal{Y})$, preserving the infinity $\mathbb{S}^{1}$ and preserving or reversing simultaneously the sense of all orbits.

We consider the six local charts $U_{i}=\left\{y \in \mathbb{S}^{2}: y_{i}>0\right\}$, and $V_{i}=\left\{y \in \mathbb{S}^{2}: y_{i}<0\right\}$ where $i=1,2,3$; and the diffeomorphisms $F_{i}: U_{i} \rightarrow \mathbb{R}^{2}$ and $G_{i}: V_{i} \rightarrow \mathbb{R}^{2}$ for $i=1,2,3$ (which are the inverses of the central projections from the planes tangent at the points $(1,0,0),(-1,0,0),(0,1,0),(0,-1,0),(0,0,1)$ and $(0,0,-1)$ respectively in order to study the sphere $\mathbb{S}^{2}$ which is a differentiable manifold). If we denote by $(u, v)$ the value of $F_{i}(y)$ or $G_{i}(y)$ for any $i=1,2,3$ (so $(u, v)$ represents different things according to the local charts under consideration), then we get for $p(\mathcal{X})$ the following expressions:

$$
\begin{array}{rr}
(3) v^{n} \Delta(u, v)\left(Q\left(\frac{1}{v}, \frac{u}{v}\right)-u P\left(\frac{1}{v}, \frac{u}{v}\right),-v P\left(\frac{1}{v}, \frac{u}{v}\right)\right) & \text { in } U_{1}, \\
(4) v^{n} \Delta(u, v)\left(P\left(\frac{u}{v}, \frac{1}{v}\right)-u Q\left(\frac{u}{v}, \frac{1}{v}\right),-v Q\left(\frac{u}{v}, \frac{1}{v}\right)\right) & \text { in } U_{2}, \\
\Delta(u, v)(P(u, v), Q(u, v)) & \text { in } U_{3},
\end{array}
$$

where $\Delta(u, v)=\left(u^{2}+v^{2}+1\right)^{-\frac{1}{2}(n-1)}$. In what follows we omit the factor $\Delta(u, v)$ by rescaling the vector field $p(\mathcal{X})$. Thus we obtain a 
polynomial vector field in each local chart. The expression for $V_{i}$ is the same as that for $U_{i}$ except for a multiplicative factor $(-1)^{n-1}$. In these coordinates for $i=1,2, v=0$ always denotes the points of $\mathbb{S}^{1}$.

2.2. Separatrices and canonical regions. Let $p(\mathcal{X})$ be the Poincaré compactification in the Poincaré disc $\mathbb{D}$ of system (1), and let $\Phi$ be its analytic flow. Following Markus [17] and Neumann [18] we denote by $(U, \Phi)$ the flow of a differential system on an invariant set $U \subset \mathbb{D}$ under the flow $\Phi$. Two flows $(U, \Phi)$ and $(V, \Psi)$ are topologically equivalent if and only if there exists a homeomorphism $h: U \rightarrow V$ which sends orbits of the flow $\Phi$ into orbits of the flow $\Psi$ either preserving or reversing the orientation of all the orbits.

A flow $(U, \Phi)$ is parallel if it is topologically equivalent to one of the following flows:

(i) The flow defined in $\mathbb{R}^{2}$ by the differential system $\dot{x}=1, \dot{y}=0$, called strip flow.

(ii) The flow defined in $\mathbb{R}^{2} \backslash\{(0,0)\}$ by the differential system in polar coordinates $\dot{r}=0, \dot{\theta}=1$, called annular flow.

(iii) The flow defined in $\mathbb{R}^{2} \backslash\{(0,0)\}$ by the differential system in polar coordinates $\dot{r}=r, \dot{\theta}=0$, called spiral or radial flow.

The separatrices of a vector field $p(\mathcal{X})$ in the Poincaré disc $\mathbb{D}$ are:

(I) all the orbits of $p(\mathcal{X})$ which are in the boundary $\mathbb{S}^{1}$ of the Poincaré disc (i.e. at the infinity of $\mathbb{R}^{2}$ ),

(II) all the finite singular points of $p(\mathcal{X})$,

(III) all the limit cycles of $p(\mathcal{X})$, and

(IV) all the separatrices of the hyperbolic sectors of the finite and infinite singular points of $p(\mathcal{X})$.

The vector fields $p(\mathcal{X})$, coming from polynomial vector fields (1) of $\mathbb{R}^{2}$ having finitely many singular points finite and infinite, have finitely many separatrices. For more details see for instance [12].

Let $\mathcal{S}$ be the union of all separatrices of the flow $(\mathbb{D}, \Phi)$ defined by $p(\mathcal{X})$ in the Poincaré disc $\mathbb{D}$. It is easy to check that $\mathcal{S}$ is an invariant closed set. If $N$ is a connected component of $\mathbb{D} \backslash \mathcal{S}$, then $N$ is also an invariant set under the flow $\Phi$ of $p(\mathcal{X})$, and the flow $\left(N,\left.\Phi\right|_{N}\right)$ is called a canonical region of the flow $(\mathbb{D}, \Phi)$.

Proposition 3. If the number of separatrices of the flow $(\mathbb{D}, \Phi)$ is finite, then every canonical region of the flow $(\mathbb{D}, \Phi)$ is parallel.

For a proof of this proposition see [18] or [12].

The separatrix configuration $\mathcal{S}_{c}$ of a flow $(\mathbb{D}, \Phi)$ is the union of all the separatrices $\mathcal{S}$ of the flow together with an orbit belonging to each 
canonical region. The separatrix configuration $\mathcal{S}_{c}$ of the flow $(\mathbb{D}, \Phi)$ is topologically equivalent to the separatrix configuration $\mathcal{S}_{c}^{*}$ of the flow $\left(\mathbb{D}, \Phi^{*}\right)$ if there exists an orientation preserving homeomorphism from $\mathbb{D}$ to $\mathbb{D}$ which transforms orbits of $\mathcal{S}_{c}$ into orbits of $\mathcal{S}_{c}^{*}$, and orbits of $\mathcal{S}$ into orbits of $\mathcal{S}^{*}$.

Theorem 4 (Markus-Neumann-Peixoto). Let $(\mathbb{D}, \Phi)$ and $\left(\mathbb{D}, \Phi^{*}\right)$ be two compactified Poincaré flows with finitely many separatrices coming from two polynomial vector fields (1). Then they are topologically equivalent if and only if their separatrix configurations are topologically equivalent.

For a proof of this result see $[17,18,20]$.

Theorem 4 implies that in order to classify the phase portraits in the Poincaré disc of a planar polynomial differential system having finitely many separatrices finite and infinite, it is enough to describe their separatrix configuration. This is what we have done in Figure 1.

\section{Phase portraits}

It is clear that system (2) with $\alpha=0$, after a rescaling of the time $\tau=(a x+b y+c) t$ becomes

$$
\begin{aligned}
& \dot{x}=y, \\
& \dot{y}=-x,
\end{aligned}
$$

whose phase portrait is formed by all the invariant circles centered at the origin of coordinates, providing the first two phase portraits of Figure 1.

In what follows we shall study the phase portraits of system (2) with $\alpha \neq 0$. Doing the rescaling of the time $\tau=\alpha t$, and renaming $a / \alpha, b / \alpha$, $c / \alpha$ again by $a, b, c$, respectively we have the quadratic system

$$
\begin{aligned}
& \dot{x}=x^{2}+y^{2}+1+y(a x+b y+c), \\
& \dot{y}=-x(a x+b y+c),
\end{aligned}
$$

with $a, b, c \in \mathbb{R}$.

Remark 5. We note that if we do the transformation $(x, y, t, c) \rightarrow$ $(-x,-y,-t,-c)$ system (5) remains invariant. Hence, it is enough to consider only the cases in which $c \geq 0$.

Remark 6. System (5) with $a=0$ is reversible because it does not change under the transformation $(x, y, t) \rightarrow(-x, y,-t)$. Hence we know that the phase portraits of system (5) with $a=0$ are symmetric with respect to the $y$-axis. 
The way for studying the phase portraits of systems (5) is the following. First we shall characterize the finite equilibria of those systems together with their local phase portraits. After we do the same for the infinite equilibria, and finally using this information on the equilibria and the existence of the invariant complex ellipse $x^{2}+y^{2}+1=0$ we shall provide the classification of all the phase portraits of systems (5).

3.1. The finite singular points. The finite singular points of system (5) are characterized in the next result.

Proposition 7. System (5) has the following finite singular points:

(a) if $b=-1$ and $c \neq 0$ then

$$
U=\left(0,-\frac{1}{c}\right)
$$

(b) if $b \neq-1$ and $c^{2}=4(b+1)$ then

$$
V=\left(0,-\frac{1}{\sqrt{b+1}}\right)
$$

(c) if $b \neq-1$ and $c^{2}>4(b+1)$ then

$$
W_{ \pm}=\left(0,-\frac{c \pm \sqrt{c^{2}-4(b+1)}}{2(b+1)}\right) .
$$

Proof. The proof follows easily studying the real solutions of the system $x^{2}+y^{2}+1+y(a x+b y+c)=0,-x(a x+b y+c)=0$.

We recall that $c \geq 0$. Now in the half plane $(b, c)$ we define the regions

$$
\begin{aligned}
& R_{1}=\{(b, c): b<-1, c \geq 0\} \\
& R_{2}=\{(b, c): b>-1, c>2 \sqrt{b+1}\} \\
& R_{3}=\{(b, c): b>-1,0 \leq c<2 \sqrt{b+1}\}
\end{aligned}
$$

the curves

$$
\begin{aligned}
& L_{1}=\{(b, c): b=-1, c \neq 0\} \\
& L_{2}=\{(b, c): b>-1, c=2 \sqrt{b+1}\}
\end{aligned}
$$

and the point $P_{1}=\{b=-1, c=0\}$.

In view of Proposition 7 , if the parameters $(b, c)$ of system (5) are in $R_{3}$ then there are no singular points. Moreover, if the parameters $(b, c)$ of system $(5)$ correspond to the point $P_{1}$ then again there are no singular points.

From now on we only consider the remaining cases: $L_{1}, L_{2}, R_{1}$ and $R_{2}$. 
For definitions of elliptic, parabolic and hyperbolic sectors, cusp, and hyperbolic, semi-hyperbolic and nilpotent singular points see [7].

We introduce some notation that will be used in the following result.

$$
\begin{aligned}
\Delta & =\sqrt{c^{2}-4(b+1)}, \quad D_{W_{+}}=-\frac{\Delta}{2(b+1)}(c(2+b)-b \Delta), \\
T_{W_{+}} & =\frac{a(-c-\Delta)}{2(1+b)}, \quad D_{W_{-}}=\frac{\Delta}{2(b+1)}(c(2+b)+b \Delta), \\
T_{W_{-}} & =\frac{a(-c+\Delta)}{2(1+b)}, \quad \operatorname{Dis}_{ \pm}=T_{W_{ \pm}}^{2}-4 D_{W_{ \pm}} .
\end{aligned}
$$

Proposition 8. System (5) has the following finite singular points if its parameters $(b, c)$ are in

$\left(L_{1}\right)$ one singular point $U$ : is a center if $a=0$; an unstable hyperbolic node if $a^{2} \geq 4 c^{2}\left(1+c^{2}\right)$ with $a<0$; a stable hyperbolic node if $a^{2} \geq 4 c^{2}\left(1+c^{2}\right)$ with $a>0$; an unstable hyperbolic focus if $a^{2}<4 c^{2}\left(1+c^{2}\right)$ with $a<0$, and a stable hyperbolic focus if $a^{2}<4 c^{2}\left(1+c^{2}\right)$ with $a>0$.

$\left(L_{2}\right)$ one singular point $V:$ is a semi-hyperbolic saddle-node if $a \neq 0$ and a nilpotent cusp if $a=0$.

$\left(R_{1}\right)$ two singular points $W_{+}$and $W_{-}: W_{+}$is a center if $a=0$; an unstable hyperbolic node if $a>0$ and Dis s $_{+} \geq 0$; a stable hyperbolic node if $a<0$ and Dis $_{+} \geq 0$; an unstable hyperbolic focus if $a>0$ and Dis $\mathrm{s}_{+}<0$ and a stable hyperbolic focus if $a<0$ and Dis + $_{+}<0 ; W_{-}$is a center if $a=0$; an unstable hyperbolic node if $a<0$ and Dis D $_{-} \geq 0$; a stable hyperbolic node if $a>0$ and Dis D $_{-} \geq 0$; an unstable hyperbolic focus if $a<0$ and Dis $_{-}<0$, and a stable hyperbolic focus if $a>0$ and Dis a $_{-}<0$.

$\left(R_{2}\right)$ two singular points $W_{+}$and $W_{-}: W_{+}$is a hyperbolic saddle, and $W_{-}$is a center if $a=0$; an unstable hyperbolic node if $a<0$ and Dis D $_{-} \geq 0$; a stable hyperbolic node if $a>0$ and Dis D $_{-} \geq 0$; an unstable hyperbolic focus if $a<0$ and Dis D $_{-}<0$, and a stable hyperbolic focus if $a>0$ and Dis D $_{-}<0$.

Proof. On the curve $L_{1}$ we have that $b=-1$ and $c \neq 0$. Clearly system (5) has the unique singular point $U$. The eigenvalues of the Jacobian matrix of system (5) at $U$ are

$$
\frac{-a+\sqrt{a^{2}-4 c^{2}\left(1+c^{2}\right)}}{2 c} \text { and } \frac{-a-\sqrt{a^{2}-4 c^{2}\left(1+c^{2}\right)}}{2 c} .
$$

If $a=0$, using the fact that system (5) is reversible with respect to the $y$-axis, and the eigenvalues of the Jacobian matrix are $\pm i \sqrt{1+c^{2}}$ we get that $U$ is a center. When $a \neq 0, U$ is a hyperbolic node if $a^{2} \geq$ 
$4 c^{2}\left(1+c^{2}\right)$. Using that $c \geq 0$, it is unstable if $a<0$ and stable if $a>0$, see for more details Theorem 2.15 of [7] where are described the local phase portraits of the hyperbolic singular points. If $a^{2}<4 c^{2}\left(1+c^{2}\right)$, since $a \neq 0$, then it is a hyperbolic focus, unstable if $a<0$ and stable if $a>0$.

On the curve $L_{2}$, we have $b>-1$ and $c=2 \sqrt{b+1}$. Clearly system (5) has the unique singular point $V$. The Jacobian matrix of system (5) at $V$ is

$$
J_{V}=\left(\begin{array}{cc}
-\frac{a}{\sqrt{1+b}} & 0 \\
-\frac{2+b}{\sqrt{1+b}} & 0
\end{array}\right) .
$$

Note that $b+2 \neq 0$ because $b>-1$. The eigenvalues of the Jacobian matrix of system (5) at $V$ are 0 and $-a / \sqrt{1+b}$.

If $a \neq 0$ then it is a semi-hyperbolic point. We will use Theorem 2.19 of [7] for studying the local phase portraits of semi-hyperbolic singular points. Doing the change of variables $x=X, y=Y-\frac{1}{\sqrt{b+1}}$ the equilibrium $V$ goes to the origin. Now the linear change of variables

$$
\left(\begin{array}{l}
u \\
v
\end{array}\right)=\left(\begin{array}{cc}
-\frac{2+b}{a} & 1 \\
1 & 0
\end{array}\right)\left(\begin{array}{l}
X \\
Y
\end{array}\right)
$$

transforms the linear part of the system into its real Jordan form. In these new variables we get the system

$$
\begin{aligned}
u^{\prime}= & -\frac{(1+b)(2+b)}{a} u^{2}-\frac{2(1+b)\left(a^{2}+(2+b)^{2}\right)}{a^{2}} u v \\
& -\frac{\left(a^{2}+(1+b)(2+b)\right)\left(a^{2}+(2+b)^{2}\right)}{a^{3}} v^{2} \\
v^{\prime}= & \frac{a}{\sqrt{1+b}} v+(1+b) u^{2}+\frac{a^{2}+2(1+b)(2+b)}{a} u v \\
& +\frac{(1+b)(2+b)^{2}+a^{2}(3+b)}{a^{2}} v^{2} .
\end{aligned}
$$

Using Theorem 2.19 of [7] we get that $V$ is a semi-hyperbolic saddlenode.

When $a=0$ the $V$ is a nilpotent singular point. We will use Theorem 3.5 of [7] for studying the local phase portraits of the nilpotent singular points. Again doing the change of variables $x=X, y=Y-\frac{1}{\sqrt{b+1}}$, and after the change $x=v, y=u$, and the rescaling of the independent 
variable $\tau=\frac{2+b}{\sqrt{1+b}} t$, we get the system

$$
\begin{aligned}
& \dot{u}=v+\frac{b \sqrt{1+b}}{2+b} u v, \\
& \dot{v}=-\frac{(1+b)^{3 / 2}}{2+b} u^{2}-\frac{\sqrt{1+b}}{2+b} v^{2} .
\end{aligned}
$$

By Theorem 3.5 of [7] the singular point $V$ is a cusp.

On the region $R_{1}$. System (5) has two singular points $W_{+}$and $W_{-}$. We use the notation $\Delta$ in (6). Note that $\Delta>0$. The Jacobian matrix of system (5) at $W_{+}$is

$$
J_{W_{+}}=\left(\begin{array}{cc}
-\frac{a(c+\Delta)}{2(1+b)} & -\Delta \\
-c+\frac{b(c+\Delta)}{2(1+b)} & 0
\end{array}\right) .
$$

The determinant of $J_{W_{+}}$is equal to $D_{W_{+}}$given in (6). Here $D_{W_{+}}>0$. The trace of $J_{W_{+}}, T_{W_{+}}$is given in (6). Note that $-c-\Delta<0$. So, $\operatorname{sign}\left(T_{W^{+}}\right)=\operatorname{sign}(a)$. By Remark 6 , if $a=0$ the system is reversible with respect to the $y$-axis and so $W_{+}$is a center. If $\mathrm{Dis}_{+} \leq 0$ (see (6)) then $W_{+}$is a hyperbolic node (stable if $a<0$ and unstable if $a>0$ ), and if Dis $_{+}<0$ then it is a hyperbolic focus (stable if $a<0$ and unstable if $a>0$ ).

The Jacobian matrix of system (5) at $W_{-}$is

$$
J_{W_{+}}=\left(\begin{array}{cc}
-\frac{a(c-\Delta)}{2(1+b)} & \Delta \\
-c-\frac{b(c+\Delta)}{2(1+b)} & 0
\end{array}\right) .
$$

The determinant is equal to $D_{W_{-}}$given in (6). Here, again $D_{W_{-}}>0$. The trace of $J_{W_{-}}$is $T_{W_{-}}$given in (6). Notice that since $\Delta>c$ then $\operatorname{sign}\left(T_{W_{-}}\right)=-\operatorname{sign}(a)$. If $a=0$ the system is reversible with respect to the $y$-axis and so $W_{-}$is a center. If $\mathrm{Dis}_{+} \leq 0($ see $(6))$ then $W_{-}$is a hyperbolic node (stable if $a>0$ and unstable if $a<0$ ), and if Dis s $_{+}<0$ then it is a hyperbolic focus (stable if $a>0$ and unstable if $a<0$ ).

On the region $R_{2}$ the study can be done in a similar way to the study in the region $R_{1}$. Again, system (5) has the two singular points $W_{+}$and $W_{-}$. The Jacobian matrix at $W_{+}, J_{W_{+}}$is given in (7) and its determinant, $D_{W_{+}}$is negative. So, $W_{+}$is a hyperbolic saddle. The Jacobian matrix at $W_{-}, J_{W_{-}}$is given in (8) and its determinant, $D_{W_{-}}$is positive. The trace of $J_{W_{-}}$is $T_{W_{-}}$. So, if $a=0$ then $W_{-}$is a center, and 
the other cases follow directly from the hyperbolic singular theorem, see Theorem 2.15 of [7].

\subsection{The infinite singular points.}

Proposition 9. The following three statements hold.

(a) If $b \neq-1$, system (5) has a pair of infinite singular points which are hyperbolic saddles if $b<-1$, and hyperbolic stable nodes if $b>-1$;

(b) If $b=-1$ and $a=0$ the infinity of system (5) is filled of singular points;

(c) If $b=-1$ and $a \neq 0$, system (5) has a pair of infinite singular points which are semi-hyperbolic. If $c>0$ they are saddle-nodes, if $c=0$ and $a<0$ they are stable nodes, and if $c=0$ and $a>0$ they are unstable nodes.

Proof. First we study the infinite singular points of the local chart $U_{1}$. System (5) in the local chart $U_{1}$ using (3) is

$$
\begin{aligned}
& \dot{u}=-a-(1+b) u-c v-a u^{2}-(1+b) u^{3}-c u^{2} v-u v^{2} \\
& \dot{v}=-\left(1+a u+(1+b) u^{2}+c u v+v^{2}\right) v .
\end{aligned}
$$

If $b \neq-1$, there is a unique infinite singular point of (9) which is $u=-a /(1+b), v=0$. The eigenvalues of the Jacobian matrix at this singular point are -1 and $-\left(a^{2}+(1+b)^{2}\right) /(1+b)$, which implies that this singular point is a hyperbolic saddle if $b<-1$, and a hyperbolic stable node if $b>-1$. Since the origin of the local chart $U_{2}$ is not a singular point, the statement (a) follows.

If $b=-1$, from the study done for system (9) there are no infinite singular points in the local chart $U_{1}$. System (5) in the local chart $U_{2}$ using (4) is

$$
\begin{aligned}
& \dot{u}=a u+c v+v^{2}+a u^{3}+c u^{2} v \\
& \dot{v}=u v(-1+a u+c v) .
\end{aligned}
$$

If $a=0$, then the infinity is filled by singular points.

If $a \neq 0$, the origin is a singular point of (10). It is easy to get that the eigenvalues of the Jacobian matrix at the origin are 0 and $a$, which implies that the origin is a semi-hyperbolic singular point. Using Theorem 2.19 of [7] we get that if $c \neq 0$ then the origin is a semi-hyperbolic saddle-node. If $c=0$ and $a<0$ the origin is a semi-hyperbolic stable node, and if $c=0$ and $a>0$ the origin is a semi-hyperbolic unstable node. 
Proof of Theorem 2. Using Propositions 8 and 9, and the existence of some first integrals, we shall obtain the global phase portraits of system (5) in Poincaré disc described in Figure 1.

For $P_{1}$ if $a \neq 0$ the phase portrait is given in 3 of Figure 1 , and if $a=0$ then it is given in the 4 of Figure 1. We note that the phase portrait 3 follows from the local study of the finite and infinite singular points done in Propositions 8 and 9 together with the Bendixson-Poincaré Theorem, see for instance [7]. The phase portrait 4 follows from the fact that on $P_{1}$ the system has the first integral $H=y^{2} /\left(1+x^{2}\right)$ and all the orbits satisfy $H=$ constant.

For $L_{1}$ if $a=0$ the phase portrait is given in 5 of Figure 1 , and if $a \neq 0$ then it is given in 6 of Figure 1. The phase portrait 5 follows from the fact that on $L_{1}$ the system has the first integral $H=(c-$ $y)^{2} /\left(1-c^{2}+x^{2}+2 c y\right)$. The phase portrait 6 follows from the local study of the finite and infinite singular points done in Propositions 8 and 9 together with the Bendixson-Poincaré Theorem.

For $L_{2}$ if $a=0$ the phase portrait is given in 7 of Figure 1, and if $a \neq 0$ then we get the phase portrait 8 of Figure 1 . Both phase portraits follow from the local study of the finite and infinite singular points done in Propositions 8 and 9 together with the Bendixson-Poincaré Theorem.

For $R_{1}$ if $a=0$ the phase portrait is given in 9 of Figure 1, and if $a \neq 0$ then we get the phase portrait 10 of Figure 1 . The phase portrait 9 follows from the fact that on $R_{1}$ the system has the first integral $H=(c+b y)^{2}\left(1+x^{2}+y^{2}\right)^{b}$. The phase portrait 10 follows from the local study of the finite and infinite singular points done in Propositions 8 and 9 together with the Bendixson-Poincaré Theorem.

For $R_{2}$ if $a=0$ the phase portrait is given in 11 of Figure 1, and if $a \neq 0$ then it is given in 12 of Figure 1 . The phase portrait 11 follows from the fact that on $R_{2}$ the system has the first integral $H=$ $(c+b y)^{2}\left(1+x^{2}+y^{2}\right)^{b}$. The phase portrait 12 follows from the local study of the finite and infinite singular points done in Propositions 8 and 9 together with the Bendixson-Poincaré Theorem.

For $R_{3}$ the phase portrait is given in 3 of Figure 1 . This completes the proof of Theorem 2 .

\section{ACKNOWLEDGEMENTS}

The first author is partially supported by a MINECO/FEDER grant MTM2013-40998-P, an AGAUR grant number 2014 SGR568, the grants 
FP7-PEOPLE-2012-IRSES 318999 and 316338, and the grant UNAB13$4 \mathrm{E}-1604$.

The second author is partially supported by FCT/Portugal through $\mathrm{UID} / \mathrm{MAT} / 04459 / 2013$.

\section{REFERENCES}

[1] J.C. Artés And J. Llibre, Quadratic Hamiltonian vector fields, J. Differential Equations 107 (1994), 80-95.

[2] J.C. Artés, J. Llibre And N. Vulpe, Complete geometric invariant study of two classes of quadratic systems, Electronic J. Differential Equations 2012, No. 09 (2012), 1-35.

[3] N.N. BAUtin, On the number of limit cycles which appear with the variation of coefficients from an equilibrium position of focus or center type, Mat. Sbornik 30 (1952), 181-196, Amer. Math. Soc. Transl. Vol. 100 (1954), 1-19.

[4] W.A. Coppel, A survey of quadratic systems, J. Differential Equations 2 (1966), 293-304.

[5] T. DATE, Classification and analysis of two-dimensional homogeneous quadratic differential equations systems, J. Differential Equations 32 (1979), 311334

[6] H. Dulac, Détermination et integration d'une certaine classe d'équations différentielle ayant par point singulier un centre, Bull. Sci. Math. Sér. (2) 32 (1908), 230-252.

[7] F. Dumortier, J. Llibre And J.C. Artés, Qualitative theory of planar differential systems, Universitext, Springer-Verlag, 2006.

[8] Yu.F. Kalin And N.I. VulPe, Affine-invariant conditions for the topological discrimination of quadratic Hamiltonian differential systems, Differential Equations 34 (1998), no. 3, 297-301.

[9] W. Kapteyn, On the midpoints of integral curves of differential equations of the first degree, Nederl. Akad. Wetensch. Verslag. Afd. Natuurk. Konikl. Nederland (1911), 1446-1457 (Dutch).

[10] W. KAPTEYN, New investigations on the midpoints of integrals of differential equations of the first degree, Nederl. Akad. Wetensch. Verslag Afd. Natuurk. 20 (1912), 1354-1365; 21, 27-33 (Dutch).

[11] N. A. Korol, "The integral curves of a certain differential equation", (in Russian), Minsk. Gos. Ped. Inst. Minsk (1973), 47-51.

[12] W. Li, J. Llibre, M. Nicolau And X. Zhang, On the differentiability of first integrals of two dimensional flows, Proc. Amer. Math. Soc. 130 (2002), 2079-2088.

[13] J. Llibre AND D. Schlomiuk, On the limit cycles bifurcating from an ellipse of a quadratic center, Discrete and Continuous Dynamical Systems-Series A 35 (2015), 1091-1102.

[14] V.A. Lunkevich And K. S. SiBIRSKII, Integrals of a general quadratic differential system in cases of a center, Differential Equations 18 (1982), 563-568.

[15] L.S. LyAGinA, The integral curves of the equation $y^{\prime}=\left(a x^{2}+b x y+c y^{2}\right) /\left(d x^{2}+\right.$ $e x y+f y^{2}$ ) (in Russian), Usp. Mat. Nauk, 6-2(42) (1951), 171-183. 
[16] L. Markus, Quadratic differential equations and non-associative algebras, Annals of Mathematics Studies, Vol 45, Princeton University Press, 1960, pp $185-213$

[17] L. Markus, Global structure of ordinary differential equations in the plane: Trans. Amer. Math Soc. 76 (1954), 127-148.

[18] D. A. Neumann, Classification of continuous flows on 2-manifolds, Proc. Amer. Math. Soc. 48 (1975), 73-81.

[19] T. A. Newton Two dimensional homogeneous quadratic differential systems, SIAM Review 20 (1978), 120-138.

[20] M.M. Peiхото, Dynamical Systems. Proccedings of a Symposium held at the University of Bahia, 389-420, Acad. Press, New York, 1973.

[21] D. Schlomiuk, Algebraic particular integrals, integrability and the problem of the center, Trans. Amer. Math. Soc. 338 (1993), 799-841.

[22] K. S. Sibirskit And N. I. Vulpe, Geometric classification of quadratic differential systems, Differential Equations 13 (1977), 548-556.

[23] E. V. VDovina, Classification of singular points of the equation $y^{\prime}=\left(a_{0} x^{2}+\right.$ $\left.a_{1} x y+a_{2} y^{2}\right) /\left(b_{0} x^{2}+b_{1} x y+b_{2} y^{2}\right)$ by Forster's method (in Russian), Differential Equations 20 (1984), 1809-1813.

[24] WeI Yin Ye AND Ye YANQIAN, On the conditions of a center and general integrals of quadratic differential systems, Acta Math. Sin. (Engl. Ser.) 17 (2001), 229-236.

[25] QIN YUAN-XUN, On the algebraic limit cycles of second degree of the differential equation $d y / d x=\sum_{0 \leq i+j \leq 2} a_{i j} x^{i} y^{j} / \sum_{0 \leq i+j \leq 2} b_{i j} x^{i} y^{j}$, Acta Math. Sinica 8 (1958), 23-35.

[26] H. ŻOŁA̧DEK, Quadratic systems with center and their perturbations, J. Differential Equations 109 (1994), 223-273.

Departament de Matemàtiques, Universitat Autònoma de Barcelona, 08193 Bellaterra, Barcelona, Catalonia, Spain

E-mail address: jllibre@mat.uab.cat

Departamento de Matemática, Instituto Superior Técnico, UniverSidAde De LisboA, 1049-001 LisboA, Portugal

E-mail address: cvalls@math.ist.utl.pt 